\title{
Real-time All-Digital Radio-over-Fiber LTE Transmission
}

\author{
Joris Van Kerrebrouck*, Laurens Breyne*, Haolin Li*, Johan Bauwelinck*, \\ Guy Torfs*, Piet Demeester* and Thomas Bohn ${ }^{\dagger}$ \\ ${ }^{*}$ Department of Information Technology (INTEC) - IDLab \\ Ghent University - imec \\ Technologiepark 15, B-9052 Zwijnaarde, Belgium \\ $\dagger$ Nokia Bell Labs \\ Lorenzstrasse 10, 70435 Stuttgart, Germany \\ Email: joris.vankerrebrouck@ugent.be
}

\begin{abstract}
Radio-over-fiber is a key-enabling-technology for next generation wireless networks. To support an increasing number of antennas, a CloudRAN approach that maximally aggregates base station functionality of the different antenna sites is the most economically feasible. To support light weight antenna units, analog radio-over-fiber is most promising. To mitigate linearity constraints of optical modulators, an all-digital transmitter can be used. In this paper, LTE transmission is demonstrated over $200 \mathrm{~m}$ OM4 multimode fiber using a VCSEL and a resonant transimpedance amplifier. The VCSEL is directly modulated with a high-speed 2-level signal generated in real-time on an FPGA. After reception of this signal, the adjacent channel power ratio was measured to be $\mathbf{- 4 5 . 6} \mathrm{dB}$. This is within the LTE specifications, proving this is a cost and power effective solution for next generation wireless networks.
\end{abstract}

\section{INTRODUCTION}

Radio-over-fiber (RoF) is seen as one of the key-enabling technologies of future wireless networks [1]-[3]. To accommodate the high throughput envisioned in $5 \mathrm{G}$ systems, different solutions are explored. The most straightforward ones follow the scaling path by reducing the cell size of the network. More disruptive approaches envision large-scale antenna systems to form a Massive multiple-input-multiple-output (MIMO) system. In the end, these antenna systems can even be distributed in its environment [4]. Beyond-5G approaches such as proposed in [5] even go further and target thousands of antennas interconnected by fiber infrastructure.

To interconnect all these antennas, the CloudRAN approach is economically the most viable. Here, all base station functions are centralized such that they can be efficiently shared over different antenna sites, interconnected with a fiber-based network. However, with the increasing amount of antennas, the cost of the remote antenna units (RAUs) should be reduced. This is a demand that is incompatible with digital RoF approaches such as Common Public Radio Interface (CPRI), which in essence transmits digital I/Q samples to an RAU. At the RAU, the optical signal is received, reconstructed using a DAC, up-converted using a locally synthesized RF carrier and amplified.

Another approach consists of directly modulating the RF signal on an optical carrier, such that an analog RoF link is obtained. In this way, the RF signal can be easily reconstructed at the antenna site using a linear transimpedance amplifier (TIA), for example a resonant TIA as described in [6]. Around the RF frequency, a photodiode is conjugately matched to a low noise amplifier (LNA). As such maximum power can be extracted from the photodector. One downside of this approach is the requirement of linear optical modulation. Compared to traditional non-return-to-zero transmission, the linearity requirements of wireless RF signals are tremendous and they keep rising every standard generation. As a result, expensive and costly optical modulators such as Mach-Zehnder Modulators (MZM) or highly linear directly modulated distributed feedback (DFB) lasers [7] are required.

To mitigate the linearity requirement, different authors [8], [9] explored how low-cost, less linear modulators, such as vertical cavity lasers (VCSELs), can still be used to transmit RF signals. Delta-Sigma modulation of the RF signal oversamples the signal and quantizes the signal to a limited amount of quantization levels (typically 2 , resulting in a singlebit stream). The quantizer is placed in a feedback system, which is configured to shape the quantization noise outside the frequency band of interest. As such very high signal-tonoise ratios can be obtained. Implementation of very highspeed delta-sigma modulators is however not trivial, as a result, most demonstrators rely on off-line generated bitstreams which are transmitted using a pulse pattern generator.

To allow real-time modulation of an all-digital RF transmitter, [10] combines delta-sigma modulation and pulsewidth modulation. In this paper, we present a real-time all-digital radio-over-fiber link consisting of a digital transmitter which modulates a VCSEL using the aforementioned algorithm and an analog RoF receiver. Thanks to the very-high speed interfaces available in modern FPGAs, a 27.5Gbps bitstream is generated containing an LTE signal at a central carrier frequency of $3.44 \mathrm{GHz}$. The output of the FPGA is directly connected to an $850 \mathrm{~nm}$ multimode VCSEL and transmitted over 200 m OM4 multimode fiber. A resonant TIA is employed at the receiving side. It is demonstrated that thanks to the combination of oversampling and noise shaping, a VCSEL can be used to transmit LTE signals over this link, providing a cheap and power efficient interconnection system for next 
generation wireless networks.

This paper is organized as follows: section II describes the used transmission system expanding both on the digital signal generation as well as on the resonant TIA used to receive the signal. Section III describes the transmission measurements and the paper is concluded in section IV.

\section{System DESCRIPTION}

To combine the benefits of digital RoF and analog RoF, we propose the system depicted in Fig. 1. The system consists of a digital modulator driving a VCSEL and a resonant optical receiver. The digital modulator takes digitally available I/Q samples and modulates them on a $3.44 \mathrm{GHz}$ central carrier frequency which is outputted using a 2-level pulse-width modulated (PWM) data stream of $27.5 \mathrm{Gbps}$. The modulator shapes the quantization noise originating from the limited amplitude levels in such a way that it falls outside the frequency band of interest. As a result, the analog signal can be recovered by simply bandpass filtering the signal. Additionally, due to the digital nature of this modulated signal, cheap non-linear optical transmitters can be used. In our system, a VCSEL is used. At the receive site, a photodiode is power matched to a low-noise amplifier at the frequency of interest forming a resonant TIA, as such, the required bandpass filter is directly integrated providing already partly the required out-of-band noise suppression. In the following paragraphs, the different components will be described in more detail.

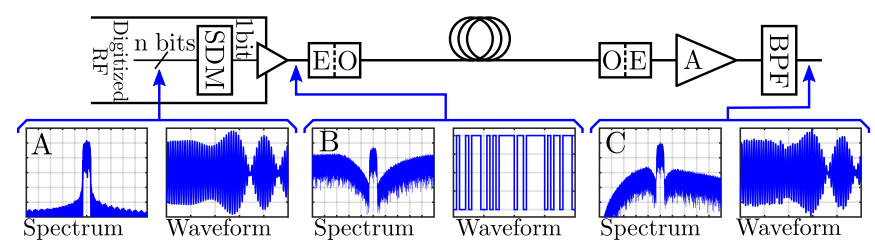

Fig. 1. Block diagram of the proposed system.

\section{A. Single-bit all-digital modulator}

There are mainly three categories of all-digital RF signal modulators. The first is pulse width modulation (PWM). The instantaneous amplitude and phase of an RF signal are mapped to a pulse width and a pulse position in a pulse train. Given the limited timing resolution of these systems, it is difficult to achieve a high signal-to-noise ratio (SNR) and meet the very stringent adjacent channel power ratio (ACPR) requirements of typical mobile communication standards. A second approach uses Delta-Sigma $(\Delta \Sigma)$ modulation [11], [12]. Here, the signal is quantized in a closed loop system. This allows to feedback the quantization noise and shapes it in such a way that inside the frequency band of interest a very high SNR can be obtained. The obtainable SNR relates to the order of the noise shaping filter and to the oversampling rate with respect to the signal bandwidth of the modulator. Excellent ACPRs can be obtained using this technique, however, the spectral efficiency is quite low and $\Delta \Sigma$ modulators are difficult to implement at very high sampling rates. A possible solution to decrease the sample rate is parallelizing $\Delta \Sigma$ modulators. But due to the non linearity in the $\Delta \Sigma$ loop, typical loop unrolling techniques used in digital signal processing can no longer be exploited. A third option is explored in [10], here a hybrid implementation combining the advantages of PWM and $\Delta \Sigma$ modulation is proposed, i.e. high coding efficiency and a good ACPR. In [10], the authors observe that the general method for encoding a complex baseband signal to a binary pulse sequence is to map the carrier frequency $\left(f_{c}\right)$ to the pulse repetition rate, the phase to the pulse position and the amplitude to the pulse width. Fig. 2 shows a single pulse with starting position $D_{a}=D_{\gamma}-\frac{1}{2} D$ and end position $D_{b}=D_{\gamma}+\frac{1}{2} D$. The complex amplitude $\left(q_{m}\right)$ of the carrier signal associated with this pulse can be calculated as follows:

$$
\begin{aligned}
q_{m} & =\int_{D_{a}}^{D_{b}} e^{-j 2 \pi f_{c} t} d t \\
& =\frac{1}{\pi} \sin (\pi D) e^{-2 \pi j D_{\gamma}}
\end{aligned}
$$

Due to the finite resolution of the PWM signal (in our case one eight of the bit period of the $\Delta \Sigma$ modulator), only a discrete set of complex baseband signals can be directly mapped to the carrier frequency. This set can be used as output signals of a complex quantizer.

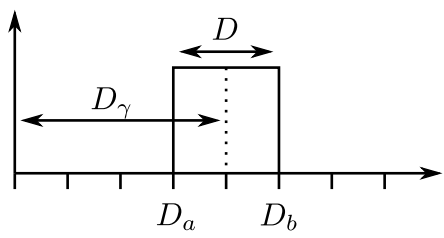

Fig. 2. Pulse position of a PWM signal.

Using this quantizer, a complex delta-sigma modulator can be constructed. The deviation from these points, which is the quantization noise, can be fed back to the input of the system such that it can be shaped outside the frequency band of interest. Fig. 3 shows the block diagram of the used modulator. The implemented modulator is first order with

$$
H(z)=\frac{z^{-1}}{1-z^{-1}}
$$

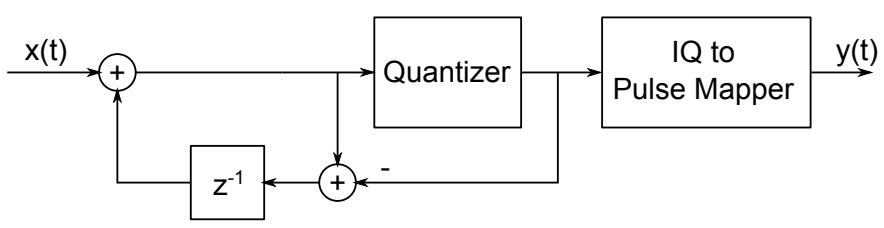

Fig. 3. Block diagram of the hybrid $\Delta \Sigma$ Pulsewidth modulator, $\mathrm{x}(\mathrm{t})$ is a multilevel complex digital baseband signal, $\mathrm{y}(\mathrm{t})$ is a 2 -level coded RF signals.

After $\Delta \Sigma$ modulator, the constellation points are then mapped to a pulse train according to their definition in (2). This process is completely implemented on an Virtex-7 FPGA and using one of the high-speed serial outputs high-speed PWM signal of 27.5 Gbps can be generated with an amplitude 
of $600 \mathrm{mV}_{\mathrm{pp}}$. The FPGA directly drives a VI Systems V50$850 \mathrm{M}, 850 \mathrm{~nm}$, multimode VCSEL. The measured electrooptical bandwidth of this device is well above $15 \mathrm{GHz}$ for driving currents exceeding $4 \mathrm{~mA}$. As a result, little bandwidth degradation will be seen when driving the VCSEL with a PWM stream of 27.5 Gbps while non-linearities of the VCSEL will be mitigated due to the single-bit modulation. The DC characteristics of the VCSEL are shown in Fig. 4. The VCSEL should be biased sufficiently high to avoid sub-threshold operation. At higher modulation currents, gain compression will occur. However, thanks to the single-bit modulation, this won't degrade the electrical to optical conversion.

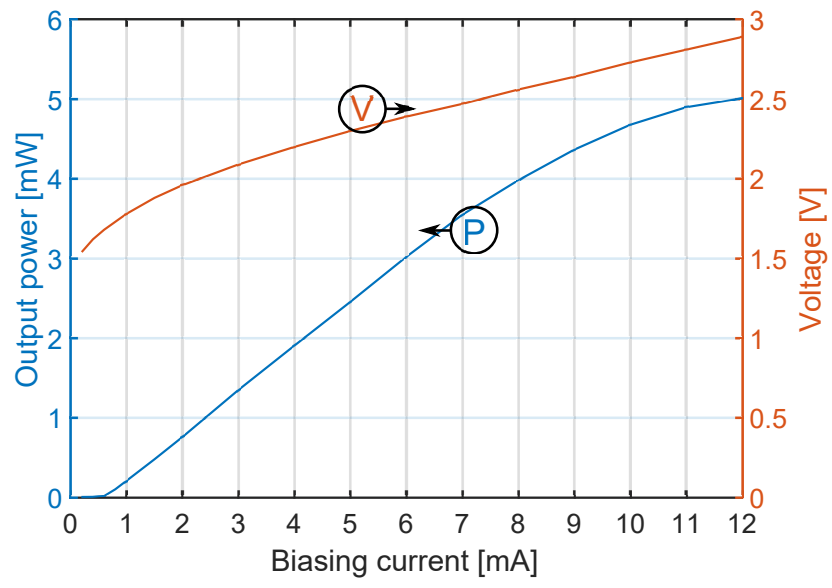

Fig. 4. Optical output power and forward voltage of the VCSEL as a function of driving current. The threshold current is $0.6 \mathrm{~mA}$ while the threshold voltage is $1.7 \mathrm{~V}$.

\section{B. Resonant TIA}

The signal is received using a resonant TIA built using a GaAs $5 \mathrm{GHz}$ PIN photodetector with a responsivity of $0.4 \mathrm{~A} / \mathrm{W}$ at $850 \mathrm{~nm}$ and a low noise amplifier. This photodetector is matched to $50 \Omega$ around $3.44 \mathrm{GHz}$. The resulting input reflection coefficient is shown in Fig. 5. Next the output of the matched photodetector is connected to a low noise amplifier (Mini-circuits, PMA3-83LN+) having $19 \mathrm{~dB}$ gain at $3.44 \mathrm{GHz}$ and an output referred $1 \mathrm{~dB}$-compression point of $18 \mathrm{dBm}$. To ensure sufficient out of band suppression an additional bandpass filter is added behind the amplifier. The complete receiver has a bandwidth of $120 \mathrm{MHz}$.

The total link, measured from the electrical input of the VCSEL, over the matched photodector, amplifier and bandpass filter, has a link budget of $4 \mathrm{~dB}$ at $3.44 \mathrm{GHz}$.

\section{MEASUREMENTS}

The goal of the experiment is to demonstrate real-time LTE transmission at $3.44 \mathrm{GHz}$ over fiber using cheap optical components such as VCSELs and multimode fiber. The photograph of the optical setup is shown in Fig. 6.

The $5 \mathrm{MHz}$ LTE test pattern used in this paper consists of 25 resource blocks, each having 12 subcarriers that are modulated with a QPSK signal, is generated. This signal

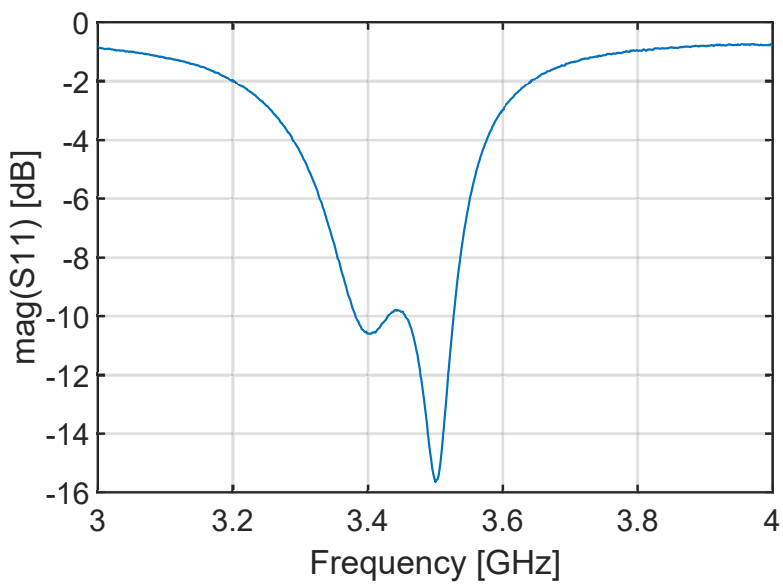

Fig. 5. Input reflection coefficient of matched photodetector.

is modulated on a single-bit stream of 27.5 Gbps with an amplitude of $600 \mathrm{mV}_{\mathrm{pp}}$. To maximize the coding efficiency, the crest factor is maximally reduced by clipping the signal, given the constraint that the error-vector-magnitude (EVM) stays within the LTE specifications. The resulting modulated stream leaving the FPGA has an in-band power of $-11.25 \mathrm{dBm}$, a crest-factor of $5.7 \mathrm{~dB}$ and an EVM of $15.16 \%$. The measured ACPR is $-49.25 \mathrm{~dB}$ which is $5 \mathrm{~dB}$ above the $-44.20 \mathrm{~dB}$ specified by the standard.

Next, the VCSEL is biased with a current of $3 \mathrm{~mA}$, yielding an optical output power of $1.4 \mathrm{dBm}$. The FPGA output is connected via a bias-tee to the modulation input of the VCSEL. The optical output is directly connecting the resonant TIA which delivers an electrical output power of $-7.25 \mathrm{dBm}$. The EVM is measured equal to the electrical measurements but the averaged ACPR is reduced to $-46.70 \mathrm{~dB}$ as shown in Fig. 7.

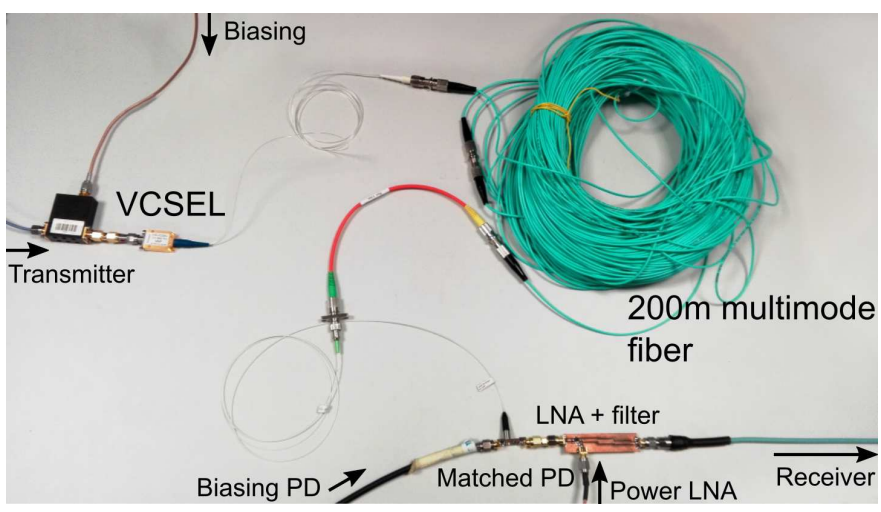

Fig. 6. Optical measurement setup.

Following, $200 \mathrm{~m}$ of OM4 multimode fiber is added between VCSEL and PD. The $200 \mathrm{~m}$ OM4 multimode fiber has an attenuation of $2.5 \mathrm{~dB} / \mathrm{km}$ and consists of 2 pieces of $100 \mathrm{~m}$. As such 2 additional fiber connections with each an attenuation of $0.4 \mathrm{~dB}$ are added, summing up to a total loss of $1.3 \mathrm{~dB}$. To compensate for the loss of the fiber, the biasing of the VCSEL 
is increased to $4 \mathrm{~mA}$ yielding an optical output power of $3 \mathrm{dBm}$. Hence, the incident optical power at the photodetector is $1.7 \mathrm{~dB}$. An attenuation of $2 \mathrm{~dB}$ in the optical domain will, due to the quadratic characteristic of the $\mathrm{PD}$, be translated to $4 \mathrm{~dB}$ of attenuation of the electrical link budget. This is also reflected in the measurements shown in Fig. 8: the received electrical power is $-11.80 \mathrm{dBm}$. The measured EVM is $15.27 \%$ and the average ACPR is $-45.6 \mathrm{~dB}$, which is still within the standard's specifications. The influence of the fiber is limited and the experiment shows that multimode fiber is suitable candidate for short-reach applications.

\begin{tabular}{|c|c|c|c|c|c|}
\hline Channel & Bandwidth & Spacing & Lower & Upper & Limit \\
\hline TX & $4.515 \mathrm{MHz}$ & & & $-7.37 \mathrm{dBm}$ & \\
\hline Adjacent & $4.515 \mathrm{MHz}$ & $5 \mathrm{MHz}$ & $-47.43 \mathrm{~dB}$ & $-45.96 \mathrm{~dB}$ & $-44.2 \mathrm{~dB}$ \\
\hline Alternate & $4.515 \mathrm{MHz}$ & $10 \mathrm{MHz}$ & $-53.62 \mathrm{~dB}$ & $-51.97 \mathrm{~dB}$ & $-44.2 \mathrm{~dB}$ \\
\hline
\end{tabular}

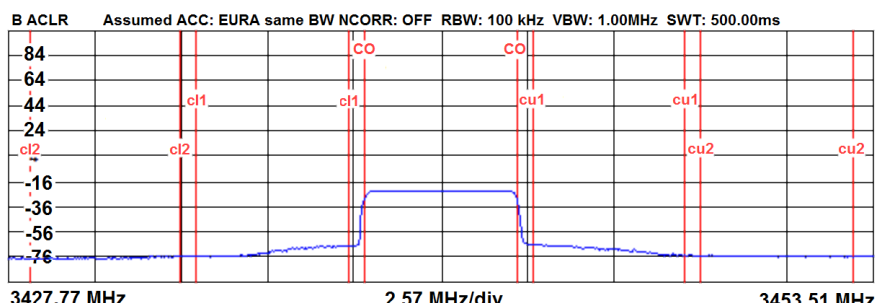

Fig. 7. Adjacent channel power ratio measurement of LTE all-digital radioover-fiber transmission back-to-back.

\begin{tabular}{|c|c|c|c|c|c|}
\hline Channel & Bandwidth & Spacing & Lower & Upper & Limit \\
\hline TX & $4.515 \mathrm{MHz}$ & & & $-11.80 \mathrm{dBm}$ & \\
\hline Adjacent & $4.515 \mathrm{MHz}$ & $5 \mathrm{MHz}$ & $-46.23 \mathrm{~dB}$ & $-44.97 \mathrm{~dB}$ & $-44.2 \mathrm{~dB}$ \\
\hline Alternate & $4.515 \mathrm{MHz}$ & $10 \mathrm{MHz}$ & $-51.38 \mathrm{~dB}$ & $-49.72 \mathrm{~dB}$ & $-44.2 \mathrm{~dB}$ \\
\hline
\end{tabular}

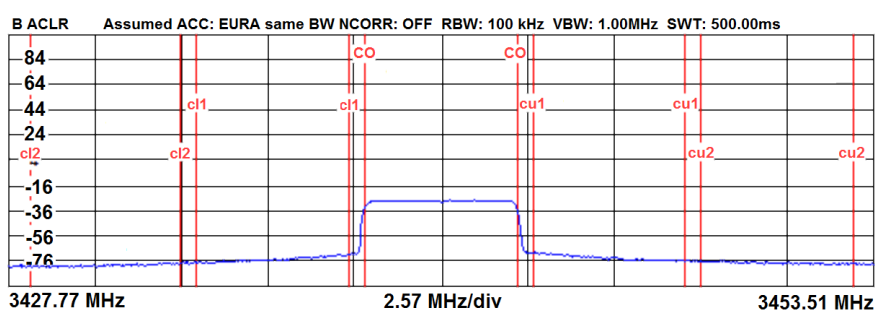

Fig. 8. Adjacent channel power ratio measurement of LTE all-digital radioover-fiber transmission over 200 m OM4 multimode fiber.

\section{CONCLUSION}

In this paper, we have demonstrated real-time all-digital radio-over-fiber transmission of LTE signals. Digital I/Q samples of an LTE signal are modulated on a $3.44 \mathrm{GHz}$ carrier using a combination of delta-sigma modulation and pulsewidth modulation. As a result, both high ACPR and coding efficiency were obtained. Thanks to the high-speed interfaces available in modern FPGAs, single-bit modulation could be used to directly drive a VCSEL mitigating its non-linear characteristic. At the receive side, a resonant TIA was used to maximize power extraction around $3.44 \mathrm{GHz}$. The demonstration verifies that LTE transmission is possible using low-cost optical components with a very lightweight remote antenna unit. The received signal undergoes minimal degradation with respect to EVM and still fulfills the adjacent channel power specifications imposed by the standard. As such, this techniques demonstrates its viability for large antenna systems to support future cellular networks.

\section{ACKNOWLEDGMENT}

This work was supported by the European Commission in the framework of the H2020-ICT-2014-2 project Flex5Gware (Grant agreement no. 671563). Piet Demeester thanks the ERC for his advanced grant 695495 ATTO: A new concept for ultrahigh capacity wireless networks.

\section{REFERENCES}

[1] P. T. Dat, A. Kanno, and T. Kawanishi, "Radio-on-radio-over-fiber: efficient fronthauling for small cells and moving cells," IEEE Trans. Wireless Commun., vol. 22, no. 5, pp. 67-75, Oct. 2015.

[2] F. Ponzini and L. Giorgi, " $5 \mathrm{G}$ radio over fiber for small-cells," in 2016 18th International Conference on Transparent Optical Networks (ICTON), Jul. 2016, pp. 1-1.

[3] B. Lannoo, A. Dixit, D. Colle, J. Bauwelinck, B. Dhoedt, B. Jooris, I. Moerman, M. Pickavet, H. Rogier, P. Simoens, G. Torfs, D. V. Ginste, and P. Demeester, "Radio-over-fibre for ultra-small 5G cells," in 2015 17th International Conference on Transparent Optical Networks (ICTON), Jul. 2015, pp. 1-4.

[4] E. Björnson, M. Matthaiou, A. Pitarokoilis, and E. G. Larsson, "Distributed massive MIMO in cellular networks: Impact of imperfect hardware and number of oscillators," in 2015 23rd European Signal Processing Conference (EUSIPCO), Aug. 2015, pp. 2436-2440.

[5] G. Torfs, H. Li, S. Agneessens, J. Bauwelinck, O. Caytan, W. Joseph, H. Rogier, A. Thielens, D. V. Ginste, X. Yin, and P. Demeester, "ATTO: Wireless networking at fiber speed," in 2017 43th European Conference and Exhibition on Optical Communications, 2017, to be published.

[6] L. Bogaert, J. Van Kerrebrouck, A. Abbasi, J. Lambrecht, G. Torfs, $\mathrm{X}$. Yin, G. Roelkens, and J. Bauwelinck, "Resonant optical receiver design by series inductive peaking for sub-6 GHz RoF," Microw. Opt. Technol. Lett., vol. 59, no. 9, pp. 2279-2284, 2017.

[7] K. Van Gasse, J. Van Kerrebrouck, A. Abbasi, G. Torfs, H. Chen, X. Yin, J. Bauwelinck, and G. Roelkens, "480Mbps / 1 Gbps radio-over-fiber link based on a directly modulated III-V-on-silicon DFB laser," in 2016 IEEE International Topical Meeting on Microwave Photonics (MWP), 2016, pp. 328-331.

[8] S. Jang, B. Park, and S. Hong, "Digital radio-over-fiber system with multi-pulse Manchester encoding-assisted delta-sigma modulation," Opt. Express, vol. 25, no. 7, pp. 8335-8349, Apr. 2017.

[9] L. M. Pessoa, J. S. Tavares, D. Coelho, and H. M. Salgado, "Experimental evaluation of a digitized fiber-wireless system employing sigma delta modulation," Opt. Express, vol. 22, p. 17508, Jul. 2014.

[10] D. Markert, X. Yu, H. Heimpel, and G. Fischer, "An All-Digital, SingleBit RF Transmitter for Massive MIMO," IEEE Trans. Circuits Syst. I, vol. 64, no. 3, pp. 696-704, Mar. 2017.

[11] Z. Ye, J. Grosspietsch, and G. Memik, "An FPGA Based All-Digital Transmitter with Radio Frequency Output for Software Defined Radio," in 2007 Design, Automation Test in Europe Conference Exhibition, Apr. 2007, pp. 1-6.

[12] A. Frappe, A. Flament, B. Stefanelli, A. Kaiser, and A. Cathelin, "An All-Digital RF Signal Generator Using High-Speed DeltaSigma Modulators," IEEE J. Solid-State Circuits, vol. 44, no. 10, pp. 2722 2732, Oct. 2009. 